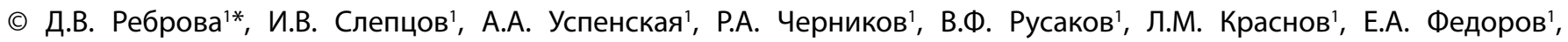
И.В. Саблин ${ }^{1}$, М.С. Ишейская ${ }^{1}$, И.В. Оловянишникова ${ }^{2}$, Ю.Н. Федотов ${ }^{1}$, А.Н. Бубнов ${ }^{1}$

1Клиника высоких медицинских технологий им. Н.И. Пирогова Санкт-Петербургского государственного университета, Санкт-Петербург, Россия

${ }^{2}$ Тольяттинская городская клиническая больница №1, Тольятти, Россия

Тиреотропинома - редкая опухоль гипофиза, являющаяся причиной развития синдрома тиреотоксикоза вследствие гиперпродукции тиреотропного гормона (ТТГ). В отечественной литературе за последние 10 лет описан один случай тиреотропиномы у ребенка, четыре случая ТТГ-продуцирующей аденомы гипофиза у женщин и только один - у мужчины. В статье представлен уникальный клинический случай 20-летней истории наблюдения больного с тиреотропиномой. Редкость данного заболевания привела к тому, что для постановки правильного диагноза потребовалось более 10 лет, даже после обнаружения сочетания аденомы гипофиза и тиреотоксикоза диагноз тиреотропиномы был установлен только через 6 лет. Категорический отказ пациента от нейрохирургической операции, непереносимость терапии аналогами соматостатина, низкий комплаенс привели к длительной персистенции тиреотоксикоза. Отсутствие зрительных дефектов, гипопитуитаризма, головных болей подтверждает данные о медленном росте ТТГ-секретирующих аденом гипофиза, даже при крупных размерах последних. Длительное влияние повышенного уровня ТТГ привело к развитию диффузного зоба с компрессией органов шеи, необходимости выполнения оперативного лечения щитовидной железы. После тиреоидэктомии у пациента с ранее диагностированным сахарным диабетом 2 типа отмечается стойкая нормогликемия, что должно привлечь внимание эндокринологов к поиску причин вторичной гипергликемии. Длительная персистенция тиреотоксикоза привела к развитию сердечной патологии в виде кардиомиопатии и фибрилляции предсердий, сохранившейся и после полного удаления щитовидной железы. Данный факт является главным аргументом в пользу своевременного радикального лечения пациентов с тиреотропиномами для устранения тиреотоксикоза и достижения стойкого эутиреоза.

КЛЮЧЕВЫЕ СЛОВА: кЛИнический случай; тиреотоксикоз; ТТГ-продуцирующая аденома гипофиза; аденома гипофиза; тиреотропинома; синдром неконтролируемой секреции ТTГ.

\title{
TSH SECRETING PITUITARY TUMOR - AN EXPERIENCE OF 20 YEARS FOLLOW-UP
}

(c) Dina V. Rebrova1*, Ilya V. Sleptsov', Anna A. Uspenskaya1, Roman A. Chernikov', Vladimir F. Rusakov', Leonid M. Krasnov'1, Elisey A. Fedorov' ${ }^{1}$ Ilya V. Sablin'1, Maria S. Isheyskaya'1, Irina V. Olovyanishnikova², Jury N. Fedotov ${ }^{1}$, Aleksandr N. Bubnov ${ }^{1}$

${ }^{1}$ Clinic of High Medical Technologies n.a. N.I. Pirogov, Saint Petersburg State University, Saint Petersburg, Russia

${ }^{2}$ Tolyatti City Clinical Hospital №1, Tolyatti, Russia

Thyrotropinoma is a rare pituitary tumor that causes the development of thyrotoxicosis syndrome as a result of hyperproduction of thyroid stimulating hormone (TSH). In the Russian literature over the past 10 years, one case of thyrotropinoma in a child, four cases of TSH-producing pituitary adenoma in women and only one in a man have been described. The article presents a unique clinical case of a 20-years history of observation of a patient with TSH-oma. The rarity of this disease led to the fact that it took more than 10 years to make a correct diagnosis. The first operation of thyroid gland was performed before the diagnosis of pituitary adenoma and inappropriate TSH secretion syndrome. That right hemithyroidectomy was supposed to cure a toxic adenoma of thyroid gland. The diagnosis of thyrotropin-secreting piruitary tumor was established only after 6 years even after finding a combination of pituitary adenoma and thyrotoxicosis. After that, the patient steadfastly refuses neurosurgical treatment, despite the presence of macroadenoma with intrasellar growth. The therapy with somatostatin analogs led to patient's intolerance with gastrointestinal side effects and hospitalization for acute pancreatitis. The absence of the therapy due to low compliance led to long-term persistence of thyrotoxicosis. The absence of signs and symptoms of expanding tumor mass (visual field defects, loss of vision, headache, partial or total hypopituitarism) demonstrates the slow growth of this kind of pituitary tumor. The long-term effect of elevated TSH levels led to diffuse goiter with compression of the neck organs, and the need of the surgical treatment of the thyroid. Stable euthyroidism after the operation led to stable normoglycemia in the patient with previously diagnosed diabetes mellitus type 2 . This fact should keep an attention of physicians and endocrinologists to screen for the secondary reasons of hyperglycemia in a patient with diabetes mellitus manifestation. Long-term history of thyrotoxicosis led to the deleterious effects of thyroid hormone excess on the heart (atrial fibrillation, cardiomyopathy, cardiac failure). Those effects are still observed even after thyroidectomy and medical euthyroidism achievement. This fact demonstrates the importance of early diagnosis and treatment of TSH-omas.

KEYWORDS: case report; TSH-secreting pituitary adenoma; pituitary adenoma; TSH; inappropriate TSH secretion syndrome. 


\section{АКТУАЛЬНОСТЬ}

Тиреотропинома - редкая опухоль, являющаяся причиной развития синдрома тиреотоксикоза вследствие избыточной продукции тиреотропного гормона (ТТГ). ТТГсекретирующие опухоли гипофиза (ТТГ-омы) представляют 0,5-1\% всех опухолей гипофиза, их распространенность составляет 1-2,8 случая на миллион жителей [1, 2]. Большинство тиреотропином являются доброкачественными (аденомы), в литературе описаны лишь единичные случаи ТТГ-продуцирующих карцином [3].

Впервые ТТГ-ома была описана в 1960 г. J.W. Jailer и D.A. Holub, которые предположили, что синдром тиреотоксикоза может быть связан с наличием опухоли гипофиза [4]. Всего с 1960 г. описано более 350 случаев заболевания [5]. В отечественной литературе за последние 10 лет описан один случай тиреотропиномы у ребенка [6], четыре случая ТТГ-продуцирующей аденомы гипофиза у женщин [7-9] и только один - у мужчины [10].

Мы представляем уникальный случай 20-летней истории наблюдения пациента с тиреотропиномой с целью расширения знаний эндокринологов о данном редком заболевании, обращения внимания на особенности течения, диагностики и дифференциальной диагностики центрального тиреотоксикоза, вторичных причин сахарного диабета.

\section{ОПИСАНИЕ СЛУЧАЯ}

Пациент Б., 65 лет, поступил в отделение эндокринной хирургии клиники высоких медицинских технологий им. Н.И. Пирогова Санкт-Петербургского государственного университета 12.04.2016 с жалобами на ощущение сдавления в области шеи, затруднение дыхания в положении лежа на спине, одышку при умеренной физической нагрузке, общую слабость, повышенную утомляемость, постоянные перебои в работе сердца.

Из анамнеза известно, что в 1995 г. впервые появились пароксизмы фибрилляции предсердий. В 1999 г. выявлен узел правой доли щитовидной железы 7×6 мм. В 2000 г. по месту жительства выполнена правосторонняя гемитиреоидэктомия по поводу узлового токсического зоба (со слов пациента, заключение гистологии «узел в щитовидной железе с признаками повышенной функциональной активности», данные не представлены). Через 3 мес после операции в связи с повышением уровня ТТГ до 68 мкMЕ/мл назначена заместительная гормональная терапия тироксином в дозе 50 мкг в сутки, которая была увеличена в связи с отсутствием нормализации уровня ТТГ до 75 мкг в сутки. На фоне приема тироксина в дозе 75 мкг в сутки появились клинические признаки тиреотоксикоза. При лабораторном обследовании установлено повышение уровня свободного тироксина (св. Т4) до 28 пмоль/л (9-22) при ТТГ 15 мкМЕ/мл $(0,4-4,0)$. Доза заместительной гормональной терапии снижена до 50 мкг в сутки. При рентгенологическом исследовании черепа выявлено увеличение турецкого седла, при магнитно-резонансной томографии (МРТ) головного мозга - макроаденома гипофиза. Уровни лютеинизирующего гормона (ЛГ), фолликулостимулирующего гормона (ФСГ), пролактина и адренокортикотропного гормона находились в пределах нормальных значений.
Макроаденома расценена как гормонально-неактивная. Учитывая размеры опухоли гипофиза, рекомендована нейрохирургическая операция, от которой пациент категорически отказался. Назначена терапия агонистами дофаминовых рецепторов (бромокриптин, далее заменен на каберголин), при приеме которых отмечались выраженная тошнота, сильная головная боль, даже на фоне приема небольших доз, в связи с чем препараты были отменены. В течение двух лет наблюдался в поликлинике по месту жительства, принимал заместительную терапию тироксином в дозе 50 мкг в сутки.

В 2002 г. госпитализирован в связи с пароксизмом фибрилляции предсердий, выявлены клинические признаки тиреотоксикоза, отменен тироксин. При дальнейшем контроле с 2002 по 2006 гг. без заместительной гормональной терапии уровень ТТГ находился в пределах 10,6-16,3 мкMЕ/мл на фоне повышенных уровней св. Т4 и свободного трийодтиронина (св. Т3).

В 2006 г. при госпитализации по месту жительства по поводу пароксизма фибрилляции предсердий выявлен тиреотоксикоз, назначена тиреостатическая терапия, по данным МРТ отмечался рост макроаденомы гипофиза. Впервые установлен диагноз тиреотропиномы. В связи с отказом пациента от нейрохирургического лечения рекомендовано оперативное лечение щитовидной железы или терапия радиоактивным йодом после достижения медикаментозного эутиреоза. Пациент от предложенных методов лечения отказался. При дальнейшем наблюдении периодически проводилась тиреостатическая терапия тирозолом в дозе 20 мг в сутки, на фоне приема которого отмечалось быстрое улучшение общего самочувствия, снижение потливости, субъективное уменьшение ощущений перебоев в работе сердца. При достижении нормальных уровней св. Т4 и св. Т3 доза тирозола постепенно снижалась до 10 мг в сутки, на фоне чего пациент чувствовал себя хорошо, однако уровни периферических тиреоидных гормонов постепенно повышались, что требовало увеличения дозировки тиреостатической терапии. При приеме тирозола в дозе 15-20 мг в сутки с достижением стойкой нормализации уровней св. Т3 и св. Т4 отмечался рост уровня ТТГ, в связи с чем пациенту вновь рекомендовалось оперативное лечение в нейрохирургическом отделении. Больной продолжал отказываться от аденомэктомии. Учитывая возможное влияние повышенного уровня ТТГ на рост тиреотропиномы, а также субъективное ощущение слабости и вялости на фоне длительного приема тирозола, тиреостатическую терапию самостоятельно отменял на 12-18 мес. В этот период отмечалось нарастание клинических симптомов, прием тирозола возобновлялся в дозе 20 мг в сутки. В дальнейшем отмена и возобновление тиреостатической терапии повторялись в схожем режиме.

С 2009 г. установлена постоянная форма мерцательной аритмии.

В 2010 г. консультирован нейрохирургом в Женеве (Швейцария), рекомендовано оперативное лечение, от которого пациент отказался. Назначена терапия аналогами соматостатина (сандостатин ЛАР 1 раз в месяц). На фоне введения препарата в течение 3 месяцев отмечал нарастание общей слабости, тошноту, снижение аппетита, уменьшение массы тела 

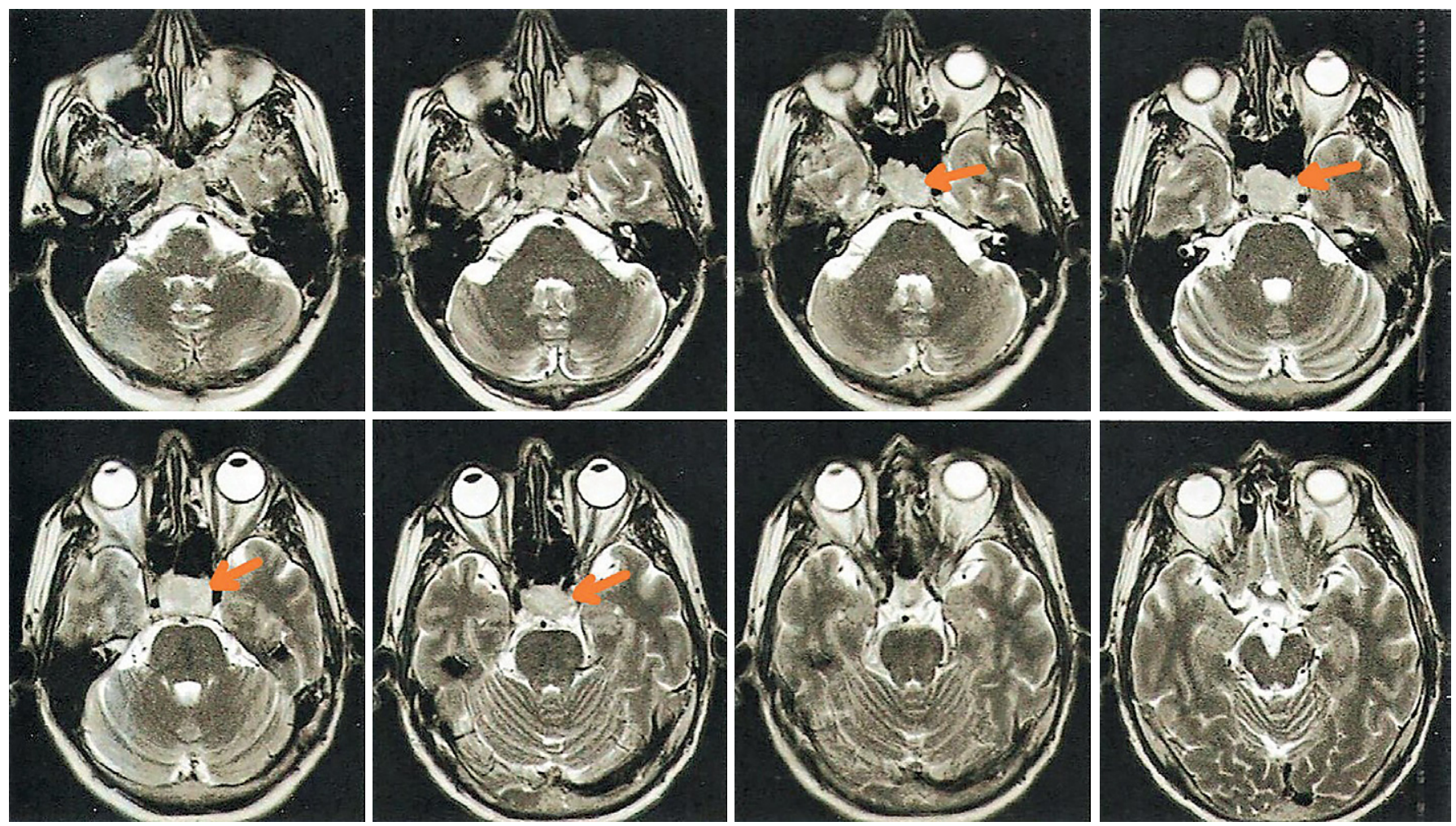

Рисунок 1. Магнитно-резонансная томография хиазмально-селлярной области, 2010 г. Стрелкой отмечена аденома гипофиза.

на 12-15 кг. Был госпитализирован по месту жительства с клинической картиной острого панкреатита, сандостатин ЛАР был отменен. В связи с развитием тиреотоксикоза, увеличением в объеме левой доли щитовидной железы, отказом пациента от оперативного лечения гипофиза в октябре 2010 г. рекомендована резекция оставшейся доли щитовидной железы, от которой пациент категорически отказался. Данные МРТ от 2010 г. представлены на рисунке 1. Также выполнена рентгенография грудного и поясничного отделов позвоночника в прямой и боковой проекциях - данных за компрессионные переломы не получено, выявлены рентгенологические признаки остеохондроза.

С 2010 по 2016 гг. тиреостатической терапии не получал, к врачу не обращался.

Настоящее ухудшение с декабря 2015 г., когда стал отмечать нарастание одышки, общей слабости, тремор рук и всего тела, ощущение внутренней дрожи, перебоев в работе сердца, прогрессивное снижение веса. От предложенного нейрохирургического лечения пациент продолжал отказываться. Направлен в Клинику высоких медицинских технологий им. Н.И. Пирогова СПбГУ с целью тиреоидэктомии. Для подготовки к операции назначена тиреостатическая терапия тирозолом 30 мг в сутки с постепенным снижением до 20 мг в сутки, на фоне приема которой отмечалось клиническое улучшение общего самочувствия пациента, в течение 6 нед достигнута нормализация уровней св. Т4 и св. Т3.

Лабораторные данные от 07.04.2016: антитела к рецепторам ТТГ 1,17 Е/л (0-2), соматотропный гормон (СТГ) 0,5 нг/мл (0-6), инсулиноподобный фактор роста-1 (ИФР-1) 199,4 нг/мл (124-310), ФСГ 5,2 мМЕ/мл (0,95-12,00), ЛГ 6,2 мМЕ/мл (1,5-9,3), тестостерон 9,33 нг/мл (8,6-23,4), ТТГ 24,2 мкMЕ/мл (0,4-4,0), кальцитонин менее 1 нг/мл (0-2), кортизол 6 мкг/дл $(3,7-19,4)$.
Сцинтиграфия щитовидной железы (от 12.02.2016): Расположение загрудинное. Рубцовая ткань правой доли 16,6×11,6 мм - накопление радиофармпрепарата (РФП) низкое. Левая доля 78,8×49,8 мм - резко увеличена в размере, включение РФП повышено, распределение неравномерно. От верхнего полюса по внутреннему краю левой доли видна добавочная доля 32,2×18,3 мм (рис. 2).

МРТ головного мозга (от 04.03.2016): макроаденома гипофиза с интраселлярным ростом без динамики от 2010 г.

Осмотрен офтальмологом: OU гипертоно-ангиосклеротическая ангиопатия сетчатки. Гиперметропический астигматизм. Поля зрения в пределах нормальных значений.

Осмотрен неврологом, нейрохирургом: противопоказаний для оперативного лечения щитовидной железы нет.

Сахарный диабет 2 типа выявлен в 2007 г. Изначально на диетотерапии, с 2010 г. получал метформин 850 мг 2 раза в день, с 2016 г. переведен на терапию алоглиптином 25 мг в сутки. При самоконтроле амбулаторно по глюкометру гликемия натощак 4-6 ммоль/л, через 2 ч после еды 7,9-10,5 ммоль/л. Гликированный гемоглобин $\left(\mathrm{HbA}_{1 \mathrm{c}}\right)$ от 07.04.2016-6,5\%.

Со слов, снижение высоты роста за жизнь на 1-2 см. Переломов в анамнезе не было. Боли в области спины отрицал. Денситометрия не выполнялась.

На момент поступления в отделение эндокринной хирургии Клиники высоких медицинских технологий им. Н.И. Пирогова СПбГУ принимал тирозол 10 мг 2 раза в день, дилтиазем 180 мг утром, пропранолол 40 мг утром, алоглиптин 25 мг утром. Принимал ривароксабан 20 мг в сутки, за 1 нед до госпитализации был переведен на непрямые антикоагулянты. 

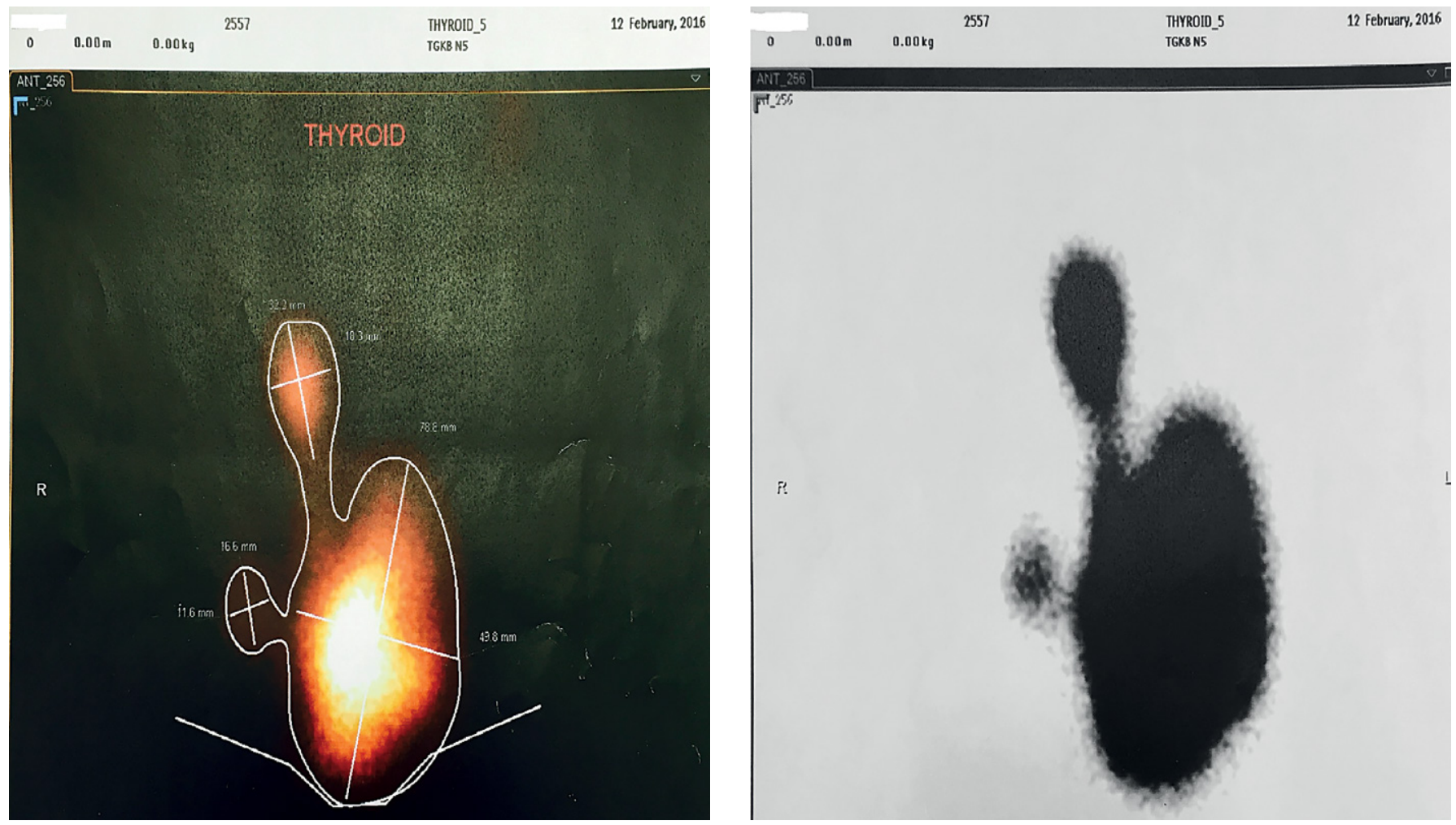

Рисунок 2. Сцинтиграфия щитовидной железы, 2016 г.

Объективно: рост 180 см, вес 70 кг, индекс массы тела 21,6 кг/м². Состояние удовлетворительное. Температура $36,7^{\circ} \mathrm{C}$. Кожные покровы чистые, естественной окраски, обычной влажности. Видимые слизистые оболочки чистые, обычной окраски. Щитовидная железа уплотнена, увеличена за счет левой доли, правая доля не пальпируется. Регионарные и периферические лимфоузлы не увеличены. Тоны сердца приглушены, аритмичные, акцент II тона над аортой, систолический шум во всех точках аускультации. Артериальное давление 130/70 мм рт. ст., пульс 84 в 1 мин, аритмичный. Число дыханий 18 в 1 мин. Дыхание везикулярное, хрипов нет. При пальпации живот мягкий, безболезненный во всех отделах. Физиологические отправления в норме. Отеков нет. Клинических признаков нарушения функции щитовидной железы, гипопитуитаризма на момент осмотра не было.

\section{Данные обследования}

В клиническом анализе крови, биохимическом анализе крови, коагулограмме, общем анализе мочи - без значимых патологических изменений. $\mathrm{HbA}_{1 с} 6,5 \%$; ТТГ 20,7 мкMЕ/мл (0,35-4,94), св. Т4 17,9 пмоль/л (9-19), св. Т3 5,6 пмоль/л (2,6-5,7); калий 3,54 ммоль/л (3,5-5,3), натрий 136,7 ммоль/л (135-148), хлориды 101 ммоль/л (97-110), кальций ионизированный 1,15 ммоль/л (1,13-1,31).

По электрокардиографии (ЭКГ) - нормосистолическая фибрилляция предсердий, 80 в 1 мин, электрическая ось сердца не отклонена. По данным суточного мониторирования ЭКГ по Холтеру все время фибрилляция предсердий с частотой сердечных сокращений 41-83-152 в 1 мин, максимальный интервал R-R 2422 мс; ишемических изменений сегмента ST нет. По эхокардиографии левое предсердие $45 \times 56$ мм, отношение конечного диастолического размера к конечному систолическому размеру левого желудочка 53/38 мм, фракция выброса 55\%, митральная недостаточность 2 степени, четких зон гипокинезии не выявлено.

МРТ хиазмально-селлярной области с контрастным усилением (рис. 3): гипофиз в турецком седле, железа увеличена в размерах до 1,9×2,0×1,7 см (поперечный,
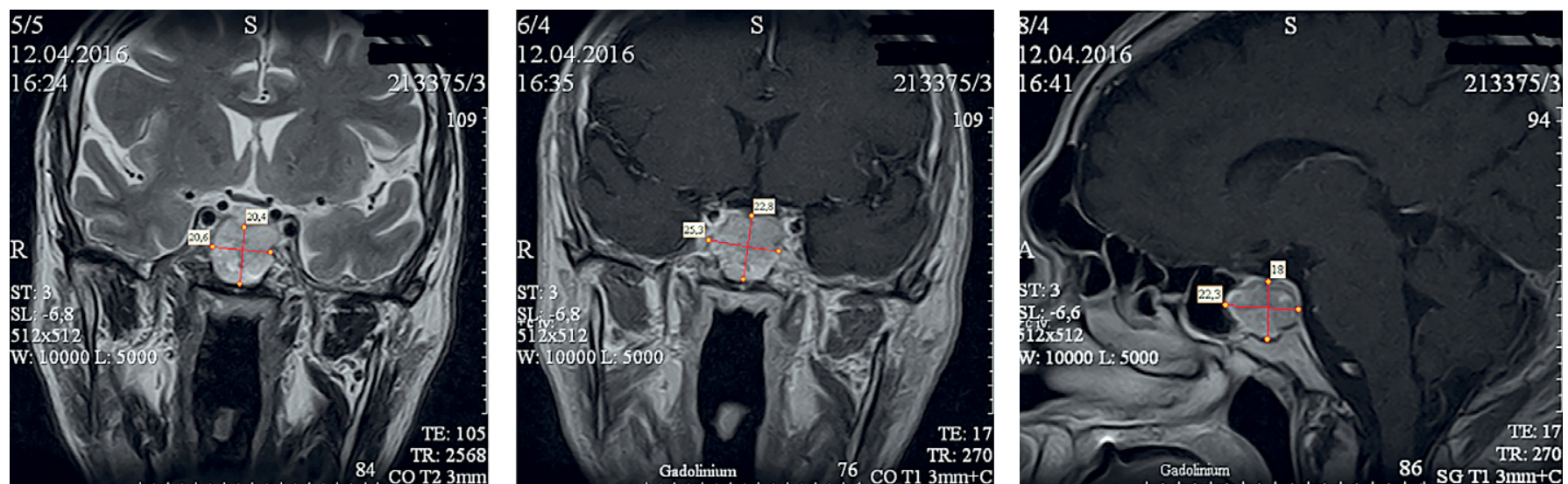

Рисунок 3. Магнитно-резонансная томография хиазмально-селлярной области, 2016 г. Выделена аденома гипофиза. 
передне-задний и вертикальный размеры соответственно) за счет образования с интраселлярным ростом. Воронка смещена вправо. Диафрагма имеет четкий контур. На постконтрастных изображениях отмечается достаточно равномерное накопление контраста данным образованием. Заключение: МРТ-признаки образования хиазмально-селлярной области (макроаденома гипофиза с интраселлярным ростом).

Ультразвуковое исследование (УЗИ) щитовидной железы: расположение железы частично загрудинное. Контуры четкие. Перешеек не лоцируется. Рубцовая ткань в ложе правой доли 10×11×17 мм (объем 0,9 мл). Левая доля 46×44×79 мм (объем 76,6 мл). Структура ткани неоднородная, с выраженными диффузными изменениями. Эхогенность ткани сниженная. Узлы не лоцируются. Лимфатические узлы не увеличены, нормальной эхоструктуры. Заключение: увеличение объема щитовидной железы, диффузные изменения структуры щитовидной железы, состояние после резекции правой доли щитовидной железы. Нижний полюс левой доли уходит за грудину.

Заключение УзИ гортани: данных о нарушении функции гортани нет.

Пересмотр CD-дисков с записью компьютерной томографии (КТ) шеи от 29.03.2016 (рис. 4): Правая доля железы удалена. Левая доля увеличена в размерах до 4,7×4,8×8,5 см. Нижний полюс левой доли щитовидной железы опускается до уровня грудинно-ключичного сочленения. Вдоль нижней поверхности подъязычной кости, по средней линии, выявляется образование
1,6×1,5×3,0 см - долька щитовидной железы? Трахея на уровне щитовидной железы деформирована, смещена вправо на 1,5 см, просвет ее не сужен (до 1,8 см).

Заключение пересмотра CD-дисков с записью КT органов грудной клетки от 29.03.2016: КТ-признаков свежих очаговых и инфильтративных изменений в легких, увеличения лимфатических узлов средостения не выявлено.

Заключение КТ органов брюшной полости и забрюшинного пространства: КТ-признаков патологических образований не выявлено.

Установлен диагноз:

Основной. ТТГ-секретирующая макроаденома гипофиза.

Осложнения. Центральный тиреотоксикоз, тяжелого течения, медикаментозный эутиреоз. Состояние после гемитиреоидэктомии справа от 2001 г. Диффузный $30 б$ II степени с загрудинной локализацией, девиацией трахеи. Дисгормональная кардиомиопатия. Постоянная форма фибрилляции предсердий, нормосистолия. Хроническая сердечная недостаточность IIA стадии, II функциональный класс (NYHA).

Сопутствующий. Сахарный диабет 2 типа. Целевой уровень $\mathrm{HbA}_{1 c}$ менее 6,5\%. Цереброваскулярная болезнь. Атеросклероз брахиоцефальных артерий без гемодинамически значимого стенозирования. Хроническое нарушение мозгового кровообращения II степени. Ишемическая болезнь сердца. Атеросклеротический кардиосклероз. Дегенеративно-кальцинированный митральный, аортальный пороки сердца, гемодинамически
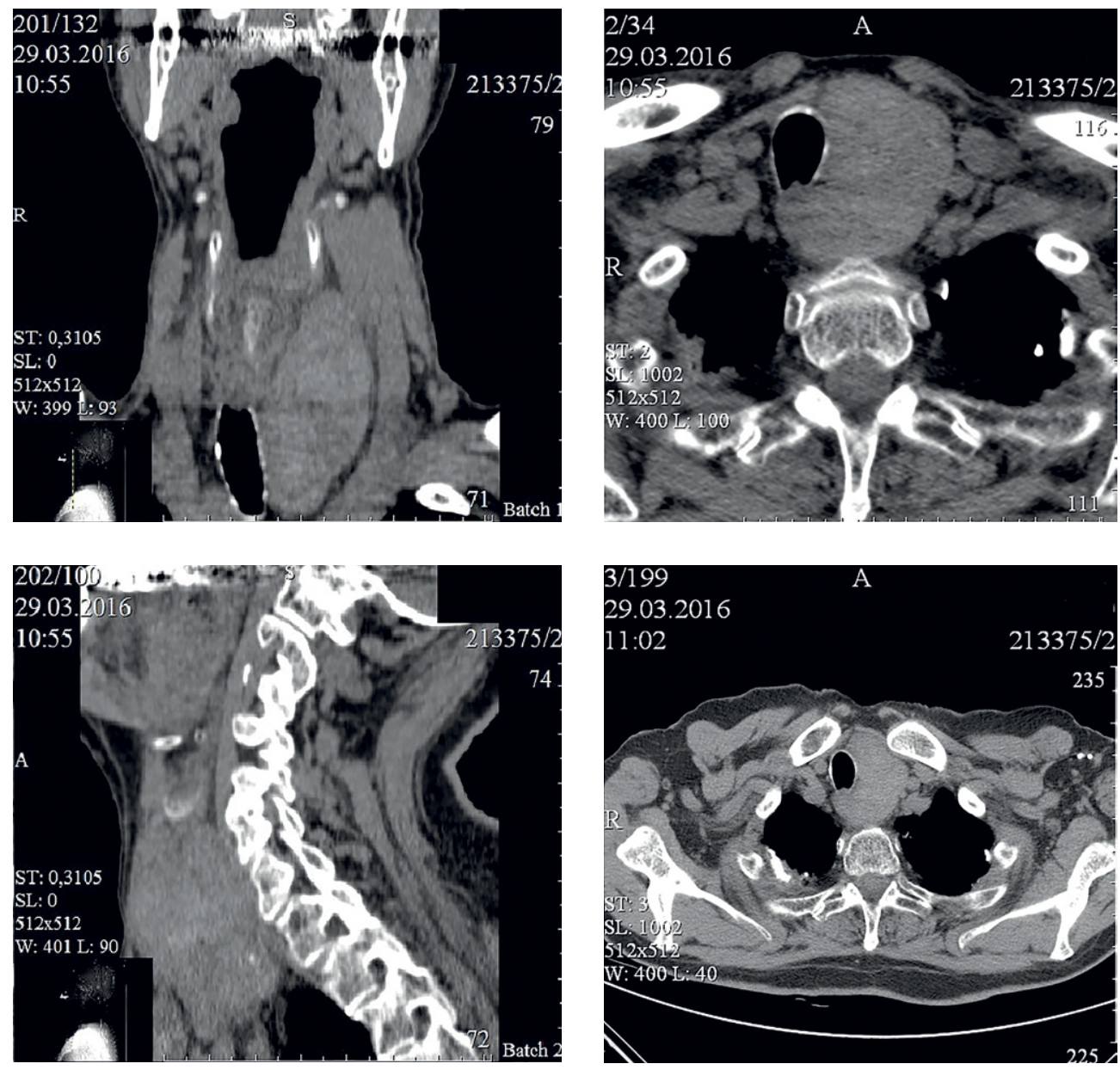

Рисунок 4. Компьютерная томография органов шеи, 2016 г. 
незначимые. Гипертоническая болезнь II стадии, артериальная гипертензия 3 степени, риск 4. Дегенеративно-дистрофическое заболевание позвоночника, преимущественно шейного отдела.

\section{Лечение}

Учитывая клинические симптомы сдавления трахеи в положении лежа на спине, загрудинное распространение диффузного зоба, категорический отказ пациента от нейрохирургического лечения, тяжелое течение центрального тиреотоксикоза, принято решение об удалении оставшейся ткани щитовидной железы.

13 апреля 2016 г. в условиях комбинированной анестезии с искусственной вентиляцией легких выполнена операция - тиреоидэктомия. Послеоперационный период протекал без осложнений. В контрольных анализах кальций ионизированный 1,14 ммоль/л $(1,13-1,31)$, паратгормон 4,0 пмоль/л (1,3-9,3). По данным контрольного УзИ гортани - голосовые связки подвижны, симметричны, смыкаются полностью. Послеоперационная рана зажила первичным натяжением.

Гистологическое исследование: макроописание доля щитовидной железы с добавочной долькой общим размером $8 \times 5 \times 3$ см; ткань доли с множественными четко очерченными серыми узлами диаметром от 0,5 до 3,5 см. Заключение: Диффузный узловой токсический зоб.

При выписке рекомендован прием препаратов: левотироксин 125 мкг в сутки, дилтиазем 240 мг утром, периндоприл 2,5 мг утром, ривароксабан 20 мг утром. Сахароснижающая терапия отменена.

\section{Дальнейшее наблюдение}

Пациент находится на амбулаторном наблюдении по месту жительства. Самочувствие хорошее. Динамика уровней тиреоидных гормонов на фоне приема заместительной терапии представлена в таблице. Через 3 мес после тиреоидэктомии доза левотироксина была уменьшена до 112,5 мкг в сутки; в 2019 г. в связи с повышенным уровнем св. Т4 при нормальной концентрации св. Т3 до 100 мкг в сутки, которую принимает по настоящее время.

На фоне полной отмены сахароснижающей терапии сохраняется нормогликемия. $\mathrm{HbA}_{1 c}$ от 08.11.2018 - 5,4\%.
От выполнения МРТ гипофиза с целью контроля роста аденомы пациент отказался, мотивировав тем, что на нейрохирургическое лечение не согласится даже при выявлении продолженного роста. При осмотре нейроофтальмолога 12.12.2018 поля зрения находятся в пределах нормальных значений, выпадения не регистрируются.

На настоящий момент больного беспокоят общая слабость, ощущения частых перебоев в работе сердца, одышка при физической нагрузке, снижение толерантности к физической нагрузке. Наблюдается кардиологом. В марте 2019 г. выполнена коронарография - данных за гемодинамически значимые стенозы коронарных артерий получено не было.

Заключение осмотра кардиолога от 02.04.2020: Кардиомиопатия смешанного генеза. Фибрилляция предсердий, постоянная форма. Пароксизмальная желудочковая тахикардия. Хроническая сердечная недостаточность IIA стадия, III функциональный класс (по классификации Нью-Йоркской ассоциации сердца). Гипертоническая болезнь 2 стадии, артериальная гипертензия 3 степени, риск сердечно-сосудистых осложнений 4. Дислипидемия. По назначению кардиолога получает лекарственную терапию: метопролол 100 мг утром, валсартан+сакубитрил 50 мг 2 раза в день, ривароксабан 20 мг утром, аторвастатин 20 мг вечером.

\section{ОБСУЖДЕНИЕ}

Тиреотропиномы в основном продуцируют изолированно ТТГ, хотя возможно и наличие ко-секреции. Чаще всего встречается сочетание гиперсекреции ТТГ и пролактина или гормона роста [11]. В связи с данным фактом, а также с целью диагностики гипопитуитаризма, вызванного сдавлением клеток гипофиза растущей опухолью, Американская ассоциация клинических эндокринологов рекомендует определение уровней пролактина, СТГ и ИФР-1, исследование гормонов надпочечников и половых желез у пациентов с выявленной ТТГ-секретирующей аденомой [12]. В представленном клиническом случае наблюдалась изолированная секреция ТТГ, остальные гормоны гипофиза находились в пределах референсных значений в течение всего времени наблюдения.

Таблица. Динамика уровней тиреоидных гормонов после удаления щитовидной железы 13.04.2016 г.

\begin{tabular}{cccc}
\hline Дата забора крови & $\begin{array}{c}\text { Уровень тиреотропного } \\
\text { гормона, мкМЕ/мл } \\
(\mathbf{0 , 4 - 4 , 0 )}\end{array}$ & $\begin{array}{c}\text { Уровень свободного } \\
\text { трийотиронина, пг/мл } \\
(\mathbf{1 , 7 - 3 , 7 )}\end{array}$ & $\begin{array}{c}\text { Уровень свободного } \\
\text { тироксина, нг/дл } \\
(\mathbf{0 , 8 - 1 , 8 )}\end{array}$ \\
\hline 14.05 .2016 & 32,99 & - & 1,11 \\
13.07 .2016 & 29,75 & 2,55 & 1,35 \\
16.09 .2016 & - & 3,01 & 1,69 \\
19.10 .2016 & - & 3,06 & 1,33 \\
15.12 .2016 & - & 2,85 & 1,40 \\
09.02 .2017 & - & 2,57 & 1,22 \\
28.05 .2018 & 20,92 & 3,13 & 1,79 \\
12.03 .2019 & 29,95 & 3,22 & 1,95 \\
30.03 .2020 & - & 2,88 & 1,46 \\
\hline
\end{tabular}


В большинстве случаев диагноз устанавливается в возрасте 50-60 лет с одинаковой частотой у мужчин и женщин [13, 14]. У пациента Б. диагноз был установлен в 55 лет. Семейные случаи тиреотропином описаны в рамках синдрома множественных эндокринных неоплазий 1 типа [15, 16], а также при семейной изолированной аденоме гипофиза в сочетании с мутациями AIP (aryl hydrocarbon receptor-interacting protein) $[17,18]$.

Диагностика тиреотропиномы основана на сочетании повышенных уровней св. Т4 и св. Т3 с неадекватно нормальным или повышенным уровнем ТТГ. Чаще всего данные лабораторные изменения сопровождаются обнаружением на МРТ опухоли гипофиза, а также отсутствием семейного анамнеза или отрицательным результатом генетического тестирования на синдром резистентности к тиреоидным гормонам (СРТГ) [12].

При выявлении подобных лабораторных данных прежде всего необходимо убедиться в отсутствии внешнего влияния на уровень тиреоидных гормонов, что может быть следствием приема некоторых лекарственных средств (амиодарон, гепарин, фуросемид), наличия антител к тиреоидным гормонам и ТТГ $[12,19]$.

При исключении методологических ошибок диагностируют синдром неконтролируемой секреции ТТГ (синдром неадекватной продукции ТТГ), включающий СРТГ и ТТГ-омы $[18,19]$. Наиболее важной представляется дифференциальная диагностика данных заболеваний в связи с тем, что лабораторные изменения при них схожи, тогда как терапевтические подходы кардинально отличаются.

СРТГ отличается аутосомно-доминантным типом наследования, в связи с чем обязательно обследование родственников первой линии для выявления схожих лабораторных данных. Характерным для СРТГ является снижение чувствительности тканей-мишеней к гормонам щитовидной железы [20]. При этом большинство больных клинически находятся в эутиреоидном состоянии, что является одним из дифференциальных критериев, так как отличительной особенностью ТТГ-секретирующих аденом гипофиза является наличие клинической картины тиреотоксикоза [12].

При смешанных опухолях, продуцирующих ТТГ и СТГ, симптомы акромегалии могут маскировать проявления тиреотоксикоза [13]. У большинства больных с тиреотропиномой в литературе описано наличие узлового или многоузлового зоба, рецидивирующего после частичных резекций щитовидной железы [14], что проявилось и в представленном случае. Развитие функциональной автономии узлов не характерно [1]. Случаи выявления дифференцированного рака щитовидной железы у данных пациентов были единичными [13].

Для дифференциальной диагностики ТТГ-секретирующих аденом гипофиза и СРТГ возможно проведение стимуляционной пробы с синтетическим аналогом тиреотропин-рилизинг-гормона, на которую тиреотропиномы дают слабый ответ с незначительным повышением уровня ТТГ $[12,14]$. Однако тиреолиберин не зарегистрирован в России, в связи с чем данный тест в настоящее время недоступен в клинической практике.

Характерным для ТТГ-секретирующих аденом является повышенная продукция а-субъединицы гликопротеидных гормонов (а-СГПГ) [18]. Американская ассоциация клинических эндокринологов рекомендует использование соотношения а-СГПГ/ТТГ в качестве диагностического критерия тиреотропиномы [12]. Однако было показано, что повышение уровня а-СГПГ выявляется не более чем у 70\% больных с макроаденомами гипофиза, для микроаденом данные изменения не характерны [16]. Кроме того, в литературе описаны различные «нормы» для данного соотношения в зависимости от пола и возраста пациентов: от 0,3 у мужчин до 29,1 у женщин в постменопаузе [21]. В связи с этим Европейская тиреоидная ассоциация не рекомендует использовать соотношение а-СГПГ/ТТГ в качестве диагностического критерия тиреотропином [14].

Надежным методом дифференциальной диагностики заболеваний, относящихся к синдрому неадекватной продукции ТТГ, является генетическое исследование, направленное на выявление мутации в гене $\beta$-изоформы рецепторов к тиреоидным гормонам. Тем не менее данная мутация выявляется лишь в 75-80\% случаев СРТГ, чаще при семейных формах $[18,14]$. Пациенту Б. генетическое исследование не выполнялось.

Наиболее простым и распространенным методом выявления опухоли гипофиза является визуализация хиазмально-селлярной области с применением КТ и МРТ [22]. Однако необходимо помнить о возможности сочетания гормонально-неактивной аденомы гипофиза с СРТГ [14]. Кроме того, микроаденомы гипофиза могут не определяться при применении визуализирующих методов исследования.

В представленном случае отмечался клинически выраженный тиреотоксикоз, в анализах выявлялось повышение уровней ТТГ и периферических гормонов (св. T4, св. T3). Семейный анамнез пациента не был отягощен по заболеваниям гипофиза и щитовидной железы.

Трудности диагностики тиреотропиномы у пациента Б. даже после обнаружения опухоли гипофиза связаны с наличием гемитиреоидэктомии в анамнезе. Появившийся в 1990-х гг. тиреотоксикоз был расценен как результат гиперпродукции тиреоидных гормонов узлом щитовидной железы. Однако, учитывая малые размеры описываемого узла (менее $1 \mathrm{~cm}$ ), наличие токсической аденомы представляется маловероятным. При контроле лабораторных анализов после гемитиреоидэктомии повышенный уровень ТТГ связывался с наличием послеоперационного гипотиреоза, а последующее развитие тиреотоксикоза - с передозировкой заместительной терапии тироксином. Так как изменения уровня ТТГ связывались с последствиями оперативного лечения щитовидной железы, выявленная макроаденома гипофиза при отсутствии изменений других тропных гормонов была расценена как гормонально-неактивная. После отказа пациента от аденомэктомии проводилось лечение препаратами из группы агонистов дофаминовых рецепторов с целью уменьшения размеров опухоли гипофиза, однако данный вид терапии был отменен в связи с плохой переносимостью даже малых доз бромокриптина и каберголина.

В представленном случае обращает на себя внимание медленный рост аденомы гипофиза. При сравнении данных МРТ от 2010 и 2016 гг., представленных на рисунках 1 и 3, не было выявлено достоверных изменений размеров тиреотропиномы. Несмотря на интраселлярный 
рост ТТГ-омы, при длительном наблюдении поля зрения пациента находятся в пределах нормальных значений. Характерным является и отсутствие симптомов гипопитуитаризма, вызванного сдавлением опухолью клеток гипофиза, продуцирующих другие тропные гормоны.

Терапия аналогами соматостатина может применяться как с лечебной целью, так и служить дополнительным критерием дифференциальной диагностики ТТГ-омы и СРТГ. У больных с тиреотропиномой отмечается положительный ответ на лечение аналогами соматостатина в виде клинического улучшения [12]. В представленном случае пациента Б. терапия аналогами соматостатина в течение 3 мес привела к уменьшению симптомов тиреотоксикоза, однако на этом фоне развились осложнения со стороны желудочно-кишечного тракта с последующей госпитализацией по поводу острого панкреатита, в связи с чем препараты данной группы были отменены.

Основным видом терапии при ТТГ-секретирующей опухоли является хирургическое удаление аденомы гипофиза. По данным исследования Malchiodi E. и соавт., из 68 пациентов, перенесших транссфеноидальную аденомэктомию, в послеоперационном периоде у 75\% наблюдалась нормальная функция щитовидной железы (из них у 58\% больных отсутствовала опухоль по данным лучевых методов исследования), у 9\% отмечалась недостаточность других гормонов гипофиза, у 3\% - возник рецидив заболевания [23].

При подготовке пациента к оперативному лечению должен быть достигнут эутиреоз. Для достижения нормального уровня тиреоидных гормонов в крови назначаются тиреостатические препараты, однако длительное применение данных препаратов не рекомендовано в связи с возможным повышением уровня ТТГ и, соответственно, стимуляцией роста аденомы [18]. Пациент Б. отмечал улучшение общего самочувствия, снижение потливости, исчезновение тремора рук и ощущения «дрожи в теле» на фоне приема тирозола. Однако при продолжительной тиреостатической терапии, даже на фоне нормальных уровней св. Т4 и св. Т3, у больного появлялись вялость, апатичность и меланхоличность, что снижало его качество жизни, так как в молодые годы, по словам пациента и его супруги, отличался активным и даже авантюрным складом характера. Кроме того, больной Б. был осведомлен о возможном влиянии дополнительного повышения уровня ТТГ на фоне приема тирозола на рост опухоли гипофиза, в связи с чем, несмотря на улучшение соматического статуса, избегал постоянной тиреостатической терапии. При назначении тирозола нормализация уровней св. Т4 и св. Т3 отмечалась в течение 4-6 нед, что позволило довольно быстро подготовить пациента с тяжелым тиреотоксикозом к тиреоидэктомии.

С целью предоперационной подготовки также возможно применение терапии аналогами соматостатина или агонистами дофамина $[12,14]$. В исследовании Socin H.V. и соавт. у 23 из 26 пациентов, получавших лечение октреотидом, уровень ТТГ снизился более чем на 50\%, у большинства из них был достигнут эутиреоз [16]. У 20-50\% больных отмечалось уменьшение размера аденомы гипофиза на фоне терапии аналогами соматостатина $[24,25]$. Rimareix F. и соавт. опубликовали данные о снижении средних уровней св. Т4 и св. Т3 на 50\% и уменьшении размеров опухоли у 6 пациентов из 7 после 3 мес лечения октреотидом [26]. Наиболее крупным на настоящий момент является исследование Fukuhara N. и соавт., в которое были включены 44 больных с тиреотропиномой. Пациенты получали терапию аналогами соматостатина в течение 1 мес в качестве предоперационной подготовки. У одного пациента препарат был отменен в связи с развитием побочных эффектов, 19 получали подкожные инъекции октреотида короткого действия, 24 - длительного действия (LAR). У 84\% больных с ТТГ-омой отмечалась нормализация св. Т4, у 61\% уменьшение размеров опухоли [27]. Тем не менее было показано, что проведение предоперационной терапии аналогами соматостатина не улучшает послеоперационный прогноз (у 63\% против 57\% не обнаруживалась опухоль по данным МРТ после хирургического лечения) [23]. По данным литературы, при лечении бромокриптином значимого уменьшения размеров тиреотропиномы зарегистрировано не было [13], супрессия уровня ТТГ носила транзиторный характер [18]. В представленном случае препараты из группы агонистов дофаминовых рецепторов назначались с целью возможного уменьшения размеров макроаденомы гипофиза на этапе до выявления гормональной активности последней. На фоне приема препаратов из данной группы пациент отмечал наличие побочных эффектов в виде тошноты и головной боли, сохраняющихся даже при назначении малых доз агонистов дофаминовых рецепторов. При этом на фоне приема бромокриптина доминировали жалобы на ежедневную тошноту разной степени интенсивности, тогда как на фоне приема каберголина - на частую головную боль. Учитывая плохую переносимость агонистов дофаминовых рецепторов, препараты данной группы были отменены.

В случаях, когда выполнение радикальной аденомэктомии невозможно, прибегают к тиреоидэктомии с последующей заместительной терапией левотироксином натрия, а для контроля роста тиреотропиномы назначается медикаментозное лечение аналогами соматостатина или лучевая терапия [19]. В исследовании Malchiodi E. и соавт. у 37\% больных, перенесших лучевую терапию на область гипофиза, отмечалось снижение уровня ТТГ, при этом у 32\% из них развился гипопитуитаризм [23].

В случае пациента Б. тиреоидэктомия была рекомендована как единственный доступный вариант устранения тяжелого тиреотоксикоза, вызывавшего развитие постоянной формы фибрилляции предсердий на фоне миокардиодистрофии. После операции клинически больной отмечал улучшение самочувствия, исчезновение ощущения внутренней дрожи, тремора рук и всего тела, повышение массы тела. Кроме того, улучшение качества жизни пациента было связано с возможностью сна в положении лежа на спине на фоне исключения сдавления трахеи диффузным зобом. Тем не менее нарушения ритма сохранились и после достижения стойкого эутиреоза. Кроме того, через 3 года после тиреоидэктомии на фоне адекватно подобранной заместительной гормональной терапии левотироксином натрия, наличие кардиомиопатии привело к появлению пароксизмов желудочковой тахикардии, нарастанию «кардиологических» жалоб больного Б., увеличению функционального класса хронической сердечной недостаточности со II до III по клас- 
сификации NYHA, снижению качества жизни пациента. Необратимый характер сердечно-сосудистых осложнений гиперпродукции тиреоидных гормонов является наиболее важным аргументом для ранней диагностики и лечения подобных пациентов, при этом эндокринологам не стоит забывать о возможности развития не только первичного, но и центрального тиреотоксикоза.

После аденомэктомии гипофиза клиническое и лабораторное обследование, включающее определение уровней ТТГ, свободных фракций тиреоидных гормонов, а также других гормонов гипофиза, следует проводить 2-3 раза на протяжении первого года после операции, а в дальнейшем - ежегодно [18]. Визуализацию гипофиза рекомендовано выполнять каждые 2-3 года или ранее, в случае выявления повышенных уровней ТТГ и тиреоидных гормонов или при возникновении клинических симптомов. При сохранении макроаденомы гипофиза необходим тщательный контроль полей зрения [12]. В связи с характерологическими особенностями представленного пациента контроль МРТ хиазмально-селлярной области не выполнялся. Учитывая медленный рост опухоли гипофиза, пациент не считает необходимым частую визуализацию последней, ограничивается консультацией офтальмолога. Выполнен контроль полей зрения, данных за выпадение последних получено не было.

Интересна особенность истории пациента Б. в отношении состояния углеводного обмена. Сахарный диабет 2 типа был установлен в 2007 г., через год после диагностики ТТГ-секретирующей аденомы гипофиза. При дальнейшем амбулаторном наблюдении, как и при поступлении в Клинику высоких медицинских технологий им. Н.И. Пирогова СПбГУ, диагноз был сохранен. Целевой уровень гликированного гемоглобина достигался пероральной сахароснижающей терапией. Длительное время пациент находился на монотерапии метформином, при ухудшении состояния и нарастании тиреотоксикоза в 2016 г. был добавлен алоглиптин. После тиреоидэктомии сахароснижающие препараты были отменены с целью дифференциальной диагностики симптоматической гипергликемии и сахарного диабета 2 типа. При дальнейшем амбулаторном наблюдении отмечается стойкая нормогликемия без терапии. Обратимость нарушений углеводного обмена свидетельствует в пользу вторичного характера гипергликемии на фоне персистирующего тиреотоксикоза. Приведенный клинический случай должен напомнить эндокринологам о необходимости исключения симптоматической гипергликемии до установления диагноза сахарного диабета.

\section{ЗАКЛЮЧЕНИЕ}

Представленный клинический случай является уникальной 20-летней историей наблюдения естественного течения заболевания у пациента с тиреотропиномой. Категорический отказ больного от нейрохирургической операции, непереносимость терапии аналогами соматостатина, низкий комплаенс привели к длительной персистенции тиреотоксикоза. Отсутствие зрительных дефектов подтверждает данные о медленном росте ТТГ-секретирующих аденом гипофиза, даже при крупных размерах последних. Длительная гиперпродукция ТТГ вызвала значительное увеличение объема щитовидной железы с развитием диффузного зоба с компрессией органов шеи, несмотря на гемитиреоидэктомию по поводу узлового зоба в анамнезе. Резекция щитовидной железы до объема тиреоидэктомии устранила сдавление трахеи, а также позволила добиться стойкого эутиреоза на фоне заместительной терапии левотироксином натрия. Однако длительная персистенция тиреотоксикоза привела к развитию тяжелых сердечно-сосудистых осложнений в виде кардиомиопатии и фибрилляции предсердий, сохранившихся и после полного удаления оставшейся части щитовидной железы. Ощущение перебоев в работе сердца стало доминирующей жалобой после устранения компрессии органов шеи. Нарушения ритма сердца носят прогрессирующий характер, на фоне постоянной формы фибрилляции предсердий наблюдаются пароксизмы желудочковой тахикардии. Пациент находится на постоянной антиаритмической, антигипертензивной, антикоагулянтной терапии под контролем кардиолога. Необратимые тяжелые сердечно-сосудистые осложнения длительной персистенции являются главным аргументом в пользу своевременного радикального лечения пациентов с ТТГ-омами для устранения тиреотоксикоза и достижения стойкого эутиреоза. Нормализация уровня гликемии после оперативного лечения щитовидной железы свидетельствует о вторичном генезе гипергликемии, связанном с повышенным уровнем тиреоидных гормонов, а не о развитии сахарного диабета 2 типа как сопутствующего заболевания.

\section{ДОПОЛНИТЕЛЬНАЯ ИНФОРМАЦИЯ}

Согласие пациента. Пациент добровольно подписал информированное согласие на публикацию персональной медицинской информации в обезличенной форме в журнале «Клиническая и экспериментальная тиреоидология».

Конфликт интересов. Авторы декларируют отсутствие явных и потенциальных конфликтов интересов, связанных с публикацией настоящей статьи.

\section{СПИСОК ЛИТЕРАТУРЫ | REFERENCES}

1. Beck-Peccoz P, Brucker-Davis F, Persani L, et al. Thyrotropinsecreting pituitary tumors. Endocr Rev. 1996;17(6):610-638. doi: 10.1210/edrv-17-6-610.

2. Onnestam L, Berinder K, Burman P, et al. National incidence and prevalence of TSH-secreting pituitary adenomas in Sweden. J Clin Endocrinol Metab. 2013;98(2):626-635. doi: 10.1210/jc.2012-3362.

3. Brown RL, Muzzafar T, Wollman R, et al. A pituitary carcinoma secreting TSH and prolactin: a non-secreting adenoma gone awry. Eur J Endocrinol. 2006;154(5):639-643. doi: 10.1530/eje.1.02141.

4. Jailer JW, Holub DA. Remission of Graves' disease following radiotherapy of a pituitary neoplasm. Am J Med. 1960;28(3):497-500. doi: 10.1016/0002-9343(60)90181-9.

5. Beck-Peccoz P, Lania A, Persani L. TSH-producing adenomas. In: Jameson JL, Degroot LJ, editors. Endocrinology. 7th ed. USA: W.B. Saunders Pub.; 2015. P. 266-274. 
6. Мазеркина Н.А., Трунин Ю.Ю., Горелышев С.К., и др. Тиреотропинома у ребенка: описание клинического случая и обзор литературы // Вопросы нейрохирургии им. Н.Н. Бурденко. — 2012. — T.76. — №5. - C. 63-69. [Mazerkina NA, Trunin II, Gorelyshev SK, et al. Thyrotropin secreting pituitary adenoma in a child: case report and literature review. Burdenko's journal of neurosurgery. 2012;76(5):63-69. (In Russ.)].

7. Пржиялковская Е.Г., Газизова Д.О., Григорьев А.Ю., и др. Тиреотропинома: трудности дифференциальной диагностики (клинический случай) // Клиническая и экспериментальная тиреоидология. - 2011. - Т.7. - №2. - С. 68-73. [Prgijalkovskaja EG, Gasisova DO, Grigoriev AYu, et al. Thyrotropinproducing adenoma: diagnostic challenges (case report). Clinical and experimental thyroidology. 2011;7(2):68-73. (In Russ.)]. doi: 10.14341/ket20117268-73

8. Карапетян А.Р., Гормолысова Е.В., Галушко Е.В. Тиреотропинома. Описание двух клинических случаев // Клиническая иэкспериментальная тиреоидология. - 2016. - Т.12. №3. - C. 37-42. [Karapetyan AR, Gormolysova EV, Galushko EV. Thyrotropin-producing adenoma. Report of two clinical cases. Clinical and experimental thyroidology. 2016;12(3):37-42. (In Russ.)]. doi: 10.14341/ket2016337-42

9. Петрик Г.Г., Космачева Е.Д., Полякова Ю.И., и др. Тиреотропинома: поздний диагноз и эффективность терапии // Проблемы эндокринологии. — 2017. — T.63. — №1. - С. 39-45. [Petrik GG, Kosmacheva ED, Polyakova UI, et al. TSH-secreting pituitary adenoma: late diagnosis and effectiveness of therapy. Problemy endokrinologii. 2017:63(1):39-45. (In Russ.)]. doi: 10.14341/probl201763139-45.

10. Белякова Н.А., Васюткова О.А., Мухина Е.Б., и др. Тиреотропинома // Лечение и профилактика. - 2018. - Т.8. №2. - C. 99-101. [Belyakova NA, Vasyutkova OA, Mukhina YeB, et al. Thyrotropinoma. Lechenie i profilaktika. 2018;8(2):99-101. (In Russ.)].

11. Mouslech Z, Somali M, Sakali AK, et al. TSH-secreting pituitary adenomas treated by gamma knife radiosurgery: our case experience and a review of the literature. Hormones (Athens). 2016;15(1):122-128. doi: 10.14310/horm.2002.1640.

12. Ross DS, Burch HB, Cooper DS, et al. American Thyroid Association Guidelines for Diagnosis and Management of Hyperthyroidism and Other Causes of Thyrotoxicosis. Thyroid. 2016;26(10):1343-1421. doi: 10.1089/thy.2016.0229.

13. Beck-Peccoz P, Persani L, Mannavola D, et al. Pituitary tumours: TSH-secreting adenomas. Best Pract Res Clin Endocrinol Metab. 2009;23(5):597-606. doi: 10.1016/j.beem.2009.05.006.

14. Beck-Peccoz P, Lania A, Beckers A, et al. 2013 European Thyroid Association guidelines for the diagnosis and treatment of thyrotropin-secreting pituitary tumors. Eur Thyroid J. 2013;2(2):76-82. doi: 10.1159/000351007.

15. Corbetta S, Pizzocaro A, Peracchi M, et al. Multiple endocrine neoplasia type 1 in patients with recognized pituitary tumours of different types. Clin Endocrinol (Oxf). 1997;47(5):507-512. doi: 10.1046/j.1365-2265.1997.3311122.x.
16. Socin HV, Chanson P, Delemer B, et al: The changing spectrum of TSH-secreting pituitary adenomas: diagnosis and management in 43 patients. Eur J Endocrinol. 2003;148(4):433-442.

doi: 10.1530/eje.0.1480433

17. Daly AF, Tichomirowa MA, Petrossians $\mathrm{P}$, et al. Clinical characteristics and therapeutic responses in patients with germ-line AIP mutations and pituitary adenomas: an international collaborative study. J Clin Endocrinol Metab. 2010;95(11):373-383. doi: 10.1210/jc.2009-2556.

18. Фадеев В.В. По материалам клинических рекомендаций Европейской тиреоидной ассоциации по диагностике и лечению ТТГ-продуцирующих опухолей гипофиза // Клиническая и экспериментальная тиреоидология. — 2016. - Т.12. — №4. C. 4-10. [Fadeev VV. Review of European Thyroid Association guidelines for the diagnosis and treatment of thyrotropinsecreting pituitary tumours. Clinical and experimental thyroidology. 2016;12(4):4-10. (In Russ.)]. doi: 10.14341/ket20164.

19. Шестакова Т.П., Комердус И.В. Диагностика редких заболеваний щитовидной железы // Русский медииинский журнал. - 2015. T.23. - №8. - C. 458-460. [Shestakova TP, Komerdus IV. Diagnostika redkikh zabolevanii shchitovidnoi zhelezy. Russkii meditsinskii zhurnal. 2015;23(8):458-460. (In Russ.)].

20. Калдымова В.А., Кияев А.В., Тюльпаков А.Н. Синдром резистентности к тиреоидным гормонам // Клиническая и экспериментальная тиреоидология. — 2013. — Т.9. — №1. — C. 51-53. [Kaldymova VA, Kiyaev AV, Tyulpakov AN. Syndromes of resistance to thyroid hormone. Clinical and experimental thyroidology. 2013;9(1):51-53. (In Russ.)]. doi: 10.14341/ket20139151-53.

21. Beck-Peccoz P, Persani L, Faglia G. Glycoprotein hormone a-subunit in pituitary adenomas. Trends Endocrinol Metab. 1992;3(2):41-45. doi: 10.1016/1043-2760(92)90041-x.

22. Nazato DM, Abucham J. Diagnosis and treatment of TSHsecreting adenomas: review of a longtime experience in a reference center. J Endocrinol Invest. 2018:41(4):447-454. doi: 10.1007/s40618-017-0770-3.

23. Malchiodi E, Profka E, Ferrante E, et al. Thyrotropin-secreting pituitary adenomas: outcome of pituitary surgery and irradiation. J Clin Endocrinol Metab. 2014;99(6):2069-2076. doi: 10.1210/jc.2013-4376.

24. Socin HV, Beck-Peccoz P, Brucker-Davis F, et al. Thyrotropinsecreting pituitary tumors. Endocr Rev. 1996;17(6):610-638. doi: 10.1210/edrv-17-6-610.

25. Wallace IR, Healy E, Cooke RS, et al. TSH-secreting pituitary adenoma: benefits of pre-operative octreotide. Endocrinol Diabetes Metab Case Rep. 2015;2015:150007. doi: 10.1530/EDM-15-0007.

26. Rimareix F, Grunenwald S, Vezzosi D, et al. Primary medical treatment of thyrotropin-secreting pituitary adenomas by first generation somatostatin analogs: a case study of seven patients. Thyroid. 2015;25(8):877-882. doi: 10.1089/thy.2015.0041.

27. Fukuhara N, Horiguchi K, Nishioka H, et al. Short-term preoperative octreotide treatment for TSH-secreting pituitary adenoma. Endocr $\mathrm{J}$. 2015;62(1):21-27. doi: 10.1507/endocrj.EJ14-0118.

\section{ИНФОРМАЦИЯ ОБ АВТОРАХ [AUTHORS INFO]}

*Реброва Дина Владимировна, кандидат медицинских наук [Dina V. Rebrova, MD, PhD]; адрес: Россия, 190103, СанктПетербург, набережная реки Фонтанки, д. 154 [address: 154 Fontanka river embankment, 190103, Saint Petersburg, Russia]; ORCID: https://orcid.org/0000-0002-7840-4174; eLibrary SPIN: 6284-9008; e-mail: endocrinology@list.ru.

Слепцов Илья Валерьевич, д.м.н. [llya V. Sleptsov, MD, PhD]; e-mail: newsurgery@yandex.ru;

ORCID: https://orcid.org/0000-0002-1903-5081; eLibrary SPIN: 2481-4331

Успенская Анна Алексеевна [Anna A. Uspenskaya, MD]; e-mail: uspenskaya_anna@mail.ru; ORCID: https://orcid.org/00000003-0396-3454

Черников Роман Анатольевич, д.м.н. [Roman A. Chernikov, MD, PhD]; e-mail: yaddd@yandex.ru; ORCID: https://orcid.org/0000-0002-3001-664X; eLibrary SPIN: 7093-1088

Русаков Владимир Федорович, к.м.н. [Vladimir F. Rusakov, MD, PhD]; e-mail: rusvf@mail.ru; eLibrary SPIN: $1345-3530$

Краснов Леонид Михайлович, д.М.н. [Leonid M. Krasnov, MD, PhD]; e-mail: krasnov.surg@mail.ru

Федоров Елисей Александрович, к.м.н. [Elisey A. Fedorov, MD, PhD]; e-mail: elick@yandex.ru; eLibrary SPIN: 5673-2633

Саблин Илья Владимирович [Ilya V. Sablin, MD]; e-mail: sablin_ilya@mail.ru; eLibrary SPIN: 5479-0942

Ишейская Мария Сергеевна [Maria S. Isheyskaya, MD]; e-mail: marya22ish@gmail.com

Оловянишникова Ирина Владимировна [Irina V. Olovyanishnikova, MD]; e-mail: irina_olov@mail.ru

Федотов Юрий Николаевич, д.м.н. [Jury N. Fedotov, MD, PhD]; e-mail: 6762525@gosmed.ru;

ORCID: https://orcid.org/0000-0003-3554-1387

Бубнов Александр Николаевич д.м.н. [Jury N. Fedotov, MD, PhD]; e-mail: 6762525@gosmed.ru 


\section{ИНФОРМАЦИЯ}

Рукопись получена: 15.05.2020. Одобрена к публикации: 13.08.2020.

\section{ЦИТИРОВАТЬ:}

Реброва Д.В., Слепцов И.В., Черников Р.А., Успенская А.А., Русаков В.Ф., Краснов Л.М., Федоров Е.А., Саблин И.В., Ишейская М.С., Оловянишникова И.В., Федотов Ю.Н., Бубнов А.Н. Тиреотропинома - опыт 20-летнего наблюдения // Клиническая и экспериментальная тиреоидология. - 2020. — Т. 16. - №2. - С. 31-41. doi: https://doi.org/10.14341/ket12430

\section{TO CITE THIS ARTICLE:}

Rebrova DV, Sleptsov IV, Chernikov RA, Uspenskaya AA, Rusakov VF, Krasnov LM, Fedorov EA, Sablin IV, Isheyskaya MC, Olovyanishnikova IV, Fedotov YN, Bubnov AN. TSH secreting pituitary tumor - an experience of 20 years follow-up. Clinical and experimental thyroidology. 2020;16(2):31-41. doi: https://doi.org/10.14341/ket12430 